\title{
Impact of early intervention and disease modification in patients with predementia Alzheimer's disease: a Markov model simulation
}

This article was published in the following Dove Press journal:

ClinicoEconomics and Outcomes Research

6 October 201I

Number of times this article has been viewed

\section{David Budd' \\ Leah C Burns ${ }^{2}$ \\ Zhenchao Guo ${ }^{2}$ \\ Gilbert L'Italien ${ }^{2,3}$ \\ Pablo Lapuerta'}

'Bristol-Myers Squibb, Princeton, NJ, USA; 'Bristol-Myers Squibb, Wallingford, CT, USA; ${ }^{3}$ Yale University School of Medicine, New Haven, CT, USA
Correspondence: David Budd Global Health Economics and Outcomes Research Department, Bristol-Myers Squibb, 100 Nassau Park Blvd, Princeton, NJ 08540, USA

Tel +l 6092523485

Fax +l 6092527440

Email david.budd@bms.com
Background: Early screenings involving biomarkers and use of potential disease-modifying therapies (DMTs) may have significant humanistic implications for treatment strategies in Alzheimer's disease.

Methods: Markov models simulated transitions of patient cohorts beginning in predementia, a hypothetical early stage of Alzheimer's disease marked by objective cognitive impairment/ memory complaints without functional impairment, and followed for 10 years. Hypothetical cohorts of 10,000 patients included those who were treated with standard of care (donepezil) upon reaching mild-moderate Alzheimer's disease, a DMT in predementia, and a DMT in mildmoderate Alzheimer's disease. Transition probabilities were based on data from the Alzheimer's Disease Neuroimaging Initiative and published clinical data, and estimated for the hypothetical DMT. In each disease stage (predementia, mild, moderate, or severe), time was computed and costs were estimated using literature review and published data, and published data provided mortality rates. The impact of screening was evaluated using positive predictive value (patients identified as predementia truly at risk for transition to dementia).

Results: Earlier treatment yielded modest gains in total life-years; however, the distribution was skewed towards milder disease. Assuming a 25\% reduction in the annual risk of progression, treating predementia patients with DMT increased life-years in predementia to mild states on average from 3.2 to 4.2, while life-years spent in moderate-to-severe Alzheimer's disease decreased from 2.6 to 2.2. Average time in the community increased from 4.4 to 5.4 years, while time in long-term care declined from 1.3 to 0.9 years. This impact grows as the advantage of the novel agent increases. Screening accuracy had significant implications for cost-effectiveness.

Conclusion: If screening can accurately identify predementia patients at risk for progression, earlier treatment with DMTs has the potential benefit to patients of prolonging time in milder disease, reducing time spent with more severe disease, increasing time in the community, and reducing time in long-term care.

Keywords: Alzheimer's disease, Markov model, disease-modifying therapy, donepezil, standard of care, predementia

\section{Introduction}

Alzheimer's disease is a fatal, neurodegenerative disorder that affects more than five million people in the US, mostly the elderly. ${ }^{1}$ The disease has an estimated worldwide prevalence of 30 million people, with an annual incidence of 4.6 million. ${ }^{2}$ Without effective treatment, this number may increase to more than 115 million by $2050 .^{3}$ Progression in patients with Alzheimer's disease typically follows a predictable course marked by a decline in behavior and function, leading to loss of independence, nursing home placement, and ultimately death. ${ }^{4}$ Patients with mild cognitive impairment 
and amyloidopathy are more likely to develop Alzheimer's disease, offering a window for therapeutic interventions that may have an impact on disease progression. ${ }^{1,5}$

The current standard of care for Alzheimer's disease is limited to symptomatic therapies which provide only temporary improvement in cognitive and behavioral symptoms and at best a temporary impact on the progression of the underlying pathology of the disease. ${ }^{6}$ These treatments include the cholinesterase inhibitors donepezil, rivastigmine, and galantamine, as well as the N-methyl-D-aspartate antagonist, memantine. ${ }^{6}$ The development of disease-modifying therapies (DMTs) is ongoing and may provide some hope for afflicted individuals. ${ }^{78}$ In addition, new screening paradigms are currently being developed with increasingly accurate predictability for the progression to dementia in patients with Alzheimer's disease, especially in earlier disease. ${ }^{9-12}$ Early screening involving biomarkers, together with the use of DMTs, may have significant humanistic implications for treatment strategies. ${ }^{9}$ The purpose of this study was to examine the impact of early screening and effective DMT, particularly in the area of slowing disease progression, on patient outcomes through the course of the disease, survival, and independence as captured by time spent in a progressively severe disease state and time spent in a home setting versus long-term institutional care.

\section{Methods}

\section{Model simulations}

A Markov model was used to simulate transitions of Alzheimer's disease patient cohorts beginning in a predementia state (defined by clinical and biomarker criteria postulated as part of the Dubois criteria ${ }^{13}$ involving memory complaint plus the presence of a biomarker, such as elevated amyloid $\beta$ or cerebrospinal fluid tau levels) and followed for 10 -year periods. ${ }^{14}$ For each endpoint, scenarios were created and run
Table I Definition of disease states for Alzheimer's disease

\begin{tabular}{ll}
\hline Disease state & MMSE range \\
\hline Predementia & $26-30$ \\
Mild AD & $21-25$ \\
Moderate AD & $10-20$ \\
Severe AD & $<10$ \\
\hline
\end{tabular}

Abbreviations: AD, Alzheimer's disease; MMSE, Mini Mental State Examination.

using computer simulations. Simulations were performed using SAS 9.1 software (SAS Institute Inc, Cary, NC). For these, the Markov models used transitions of patient cohorts, with estimated transition probabilities based on published clinical data ${ }^{15}$ for patients with mild, moderate, and severe Alzheimer's disease. Data from the Alzheimer's Disease Neuroimaging Initiative ${ }^{16}$ were used for estimates of progression from predementia to Alzheimer's disease and estimates of the impact of the hypothetical DMT. For each scenario, hypothetical cohorts of 10,000 patients were created and then allowed to flow through the course of disease from entry. In the model, six-month cycles were used to estimate the occurrences of various events, ie, disease-state transition, movement from home to long-term care, and mortality. Therefore, at each six-month point, patients could remain in the current state of disease, progress to the next more severe disease state, or exit the model via death. Time in each disease state (predementia, mild, moderate, or severe) was computed. This model assumed that all patients are at risk to progress, and the relative rate of progression for each individual in each cohort was based on the transition probabilities. Each state was defined by the Mini Mental State Examination range (Table 1). ${ }^{15}$ Once patients reached a severe Alzheimer's disease state, they were removed from active treatment, whether receiving the hypothetical DMT or standard of care. The flow of the model is shown in Figure 1.

For the purpose of this exercise, the three hypothetical cohorts of 10,000 patients each were created with the following characteristics: cohort 1 (standard of care),

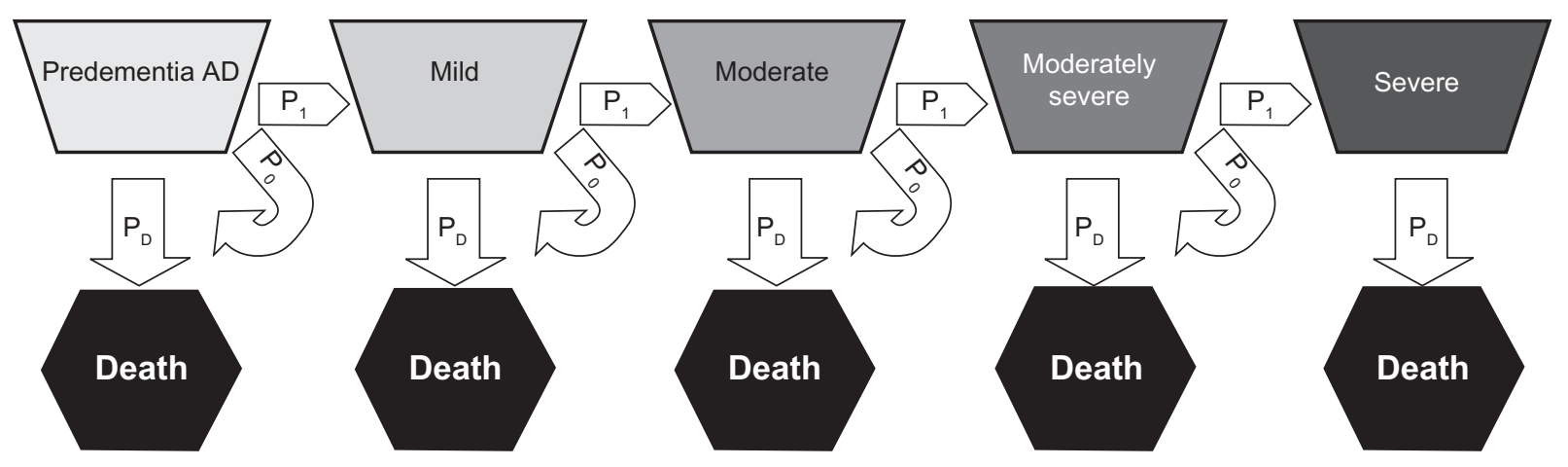

Figure I Model flow. Transition probabilities: $P_{0}$, probability of remaining in current state; $P_{1}$, probability of transitioning to next, more severe state; $P_{D}$, probability of death. Abbreviation: AD, Alzheimer's disease. 


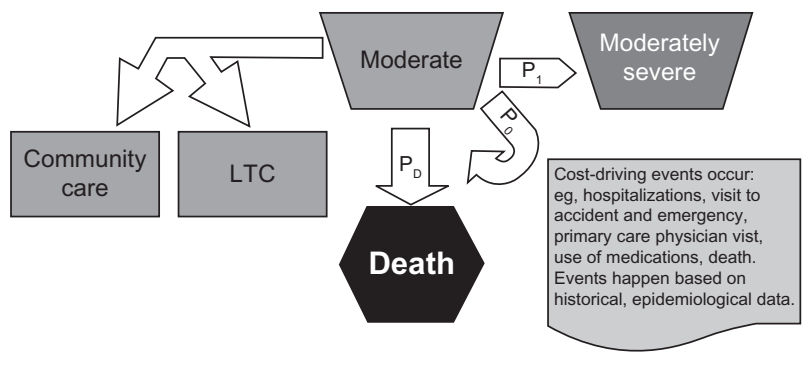

Figure 2 Intradisease state outcome details. Transition probabilities: $P_{0}$, probability of remaining in current state; $P_{1}$, probability of transitioning to next, more severe state; $P_{D}$, probability of death.

Abbreviation: LTC, long-term care.

patients treated with donepezil upon reaching mild-moderate Alzheimer's disease; cohort 2 (predementia state with DMT), patients treated with a hypothetical DMT initiated in the predementia state; and cohort 3 (mild-moderate state DMT), patients treated with a hypothetical DMT upon reaching mild Alzheimer's disease but not before. In addition, each model had three per-patient and cumulative primary endpoints: time spent in each of the four disease states (predementia, mild, moderate, and severe Alzheimer's disease); time spent living in a community/home setting versus time spent in long-term care, defined as a skilled nursing facility, nursing home, or hospital/hospice; and total life-years gained.

\section{Outcomes}

While in each state, patients could either enter long-term care or remain in the community, based on risk. The risk for entry into long-term care was defined as a function of age and disease state, based on the published literature ${ }^{17}$ (ie, the risk of placement into long-term care increases with age and disease severity).
For example, risk of placement in long-term care was much higher $(86 \%)$ in severe Alzheimer's disease compared with mild-moderate Alzheimer's disease (17\%). ${ }^{17}$ The time in each disease state (predementia, mild, moderate, moderately severe, or severe) was computed. The design of the outcome model within each disease state is shown in Figure 2.

\section{Death}

Mortality used in the model was based on all-cause mortality, according to published data from the United States Statistical Abstract 2006. ${ }^{18}$ All patients were entered into the simulation with an age of 75 years. The likelihood/ risk of death was dependent on age, gender, and the stage of Alzheimer's disease. ${ }^{19}$ Older patients had an increasing risk of death and patients with more severe disease states in Alzheimer's disease were at greater risk of death. Compared with a matched cohort of men, women of equal age and severity of disease had less risk of death. ${ }^{19}$ The baseline risk used for all-cause mortality among men in this population rose from $3.5 \%$ (aged 75 years) to $10.3 \%$ (aged 85 years) to $38.9 \%$ (aged 100 years). For women, the numbers employed were $2.5 \%, 8.2 \%$, and $38.0 \%$, respectively. The impact of disease severity on mortality was estimated by increasing the baseline mortality as a function of relative risk. Relative risk estimates were derived from the work of Neumann et al. ${ }^{19}$ For predementia and mild disease states, the relative risk for death used was 1.0 (risk of mortality equal to baseline of otherwise healthy, age-matched patients). For patients categorized with moderate Alzheimer's disease, the relative risk used was 2.52, and for patients with severe Alzheimer's disease, the relative risk used was 7.3.
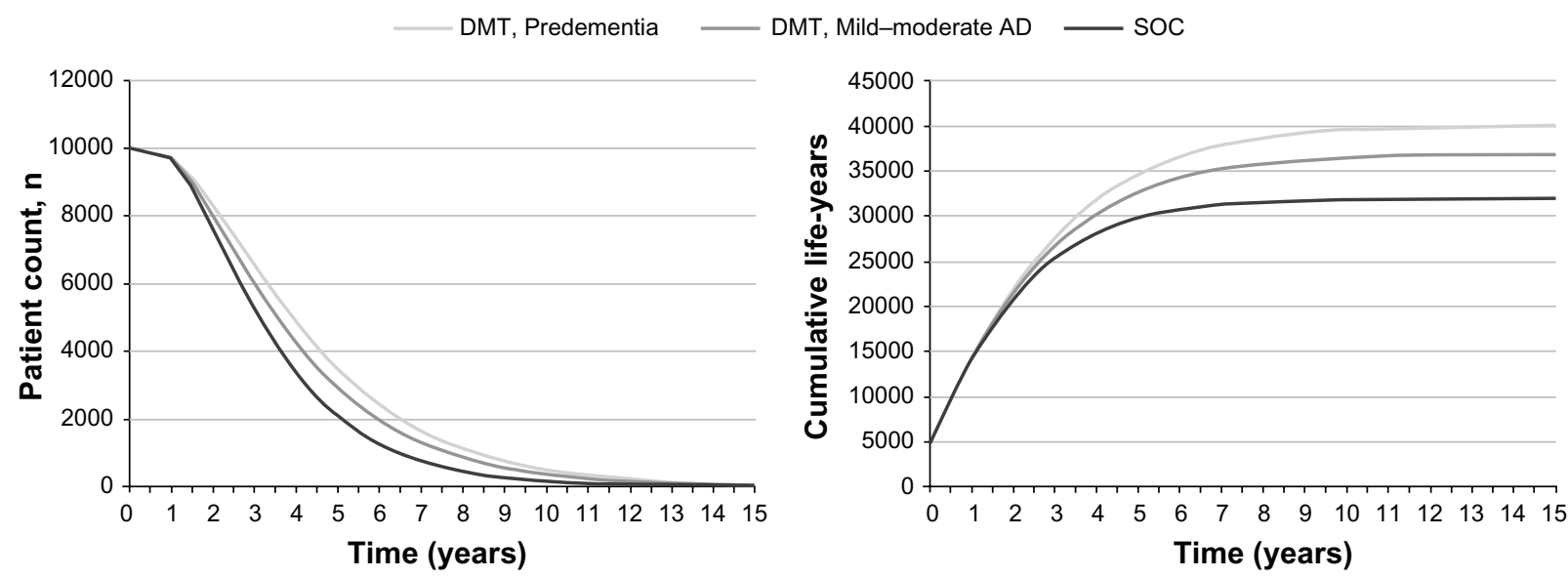

Figure 3 Left panel: Markov model results showing the estimated count of patients ( $y$ axis) and time spent (x axis) with predementia or mild AD when initiating DMT during predementia AD (light gray line), DMT during mild-moderate AD (medium gray line), or SOC during mild-moderate AD (black line). Right panel: the cumulative time spent in predementia or mild AD if initiating DMT during predementia AD (light gray line), DMT during mild-moderate AD (medium gray line), or SOC during predementia $A D$ (black line).

Abbreviations: AD, Alzheimer's disease; DMT, disease-modifying therapy; SOC, standard of care. 


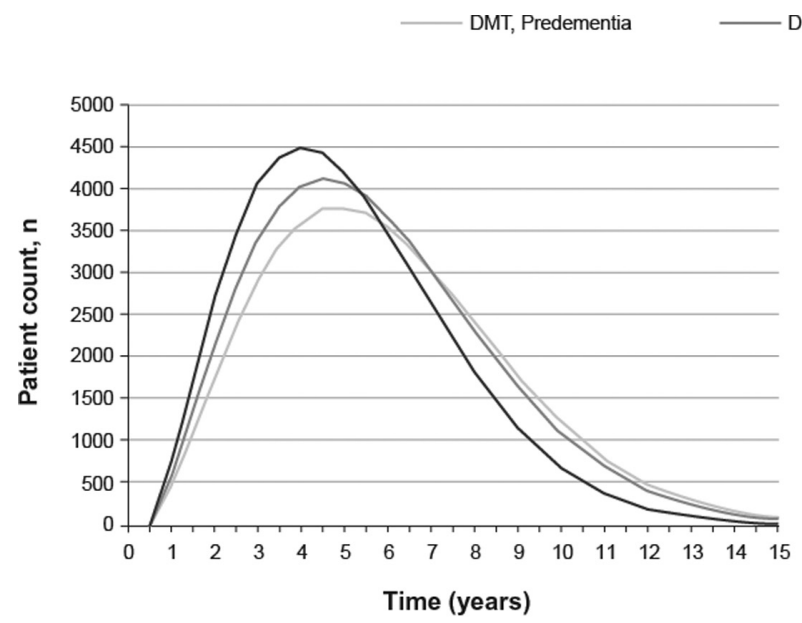
DMT, Mild-moderate AD $\quad$ SOC

Figure 4 Left panel: Markov model results showing the estimated count of patients ( $y$ axis) and time spent (x axis) with moderate-severe AD when initiating DMT during predementia AD (light gray line), DMT during mild-moderate AD (medium gray line), or SOC during mild-moderate AD (black line). Right panel: the cumulative time spent in moderate or severe AD if initiating DMT during predementia AD (light gray line), DMT during mild-moderate AD (medium gray line), or SOC during predementia AD (black line).

Abbreviations: AD, Alzheimer's disease; DMT, disease-modifying therapy; SOC, standard of care.

\section{Impact of disease modification on disease progression}

Finally, the impact of disease modification on disease progression was estimated in the three cohorts under each scenario, ie, standard of care (treatment with donepezil during mild-moderate disease), treatment during predementia Alzheimer's disease with DMT, and treatment during mildmoderate disease with DMT. As a baseline, it was assumed that the standard of care had no impact on the underlying course of disease. Transition probabilities were then based on the natural progression of disease. ${ }^{15}$ The relative effectiveness of the potential DMT was then allowed to range from $10 \%$ to $30 \%$ superior to the natural course of disease. For example, if a DMT had a 10\% superiority, the program reduced the annual rates of progression by $10 \%$ (ie, if progression from mild to moderate Alzheimer's disease were assumed to be $20 \%$ in the natural course, a $10 \%$ superiority reduced the progression rate from $20 \%$ to $18 \%$ ).

\section{Results and discussion}

This is the first analysis to explore the potential impact of early intervention with DMTs on the progression and outcomes of Alzheimer's disease. This Markov model evaluation of the impact of early intervention with DMTs in patients with Alzheimer's disease demonstrated that under all scenarios there was a modest advantage in lifeyears gained for a hypothetical DMT (Figures 3-5). The presumed relative efficacy for these plots was $25 \%$ (ie, $25 \%$ reduction in progression rates over the standard of care). Assuming that the availability of such an agent to the population could successfully slow the progression of disease, this analysis suggests that patients would gain an average of slightly less than one year of life. This is largely due to the reduced risk of mortality in milder versus more severe disease. As patients avoided more severe states, their risk of death related to Alzheimer's disease decreased. However, patients in the model were assumed to enter with an age of 75 years. This relatively elderly age at entry has a significant impact on estimated longevity, even in a healthy population. In addition, because earlier intervention with DMTs arrests the progression of disease sooner, patients in the model receiving treatment in the predementia Alzheimer's stage had a slightly longer life expectancy than those who waited for therapy until affected by moderate disease.

Table 2 Average life-years in disease states for different DMT efficacies (all data in years)

\begin{tabular}{|c|c|c|c|c|c|c|}
\hline \multirow[t]{2}{*}{ DMT relative advantage } & \multicolumn{2}{|c|}{ DMT during predementia } & \multicolumn{2}{|c|}{ DMT during moderate $A D$} & \multicolumn{2}{|c|}{ SOC during moderate AD } \\
\hline & Mild & Severe & Mild & Severe & Mild & Severe \\
\hline $10 \%$ & 3.46 & 2.54 & 3.35 & 2.60 & 3.19 & 2.56 \\
\hline $20 \%$ & 3.78 & 2.40 & 3.54 & 2.53 & 3.19 & 2.56 \\
\hline $25 \%$ & 3.96 & 2.32 & 3.65 & 2.49 & 3.19 & 2.56 \\
\hline
\end{tabular}

Abbreviations: AD, Alzheimer's disease; DMT, disease-modifying therapy; SOC, standard of care. 


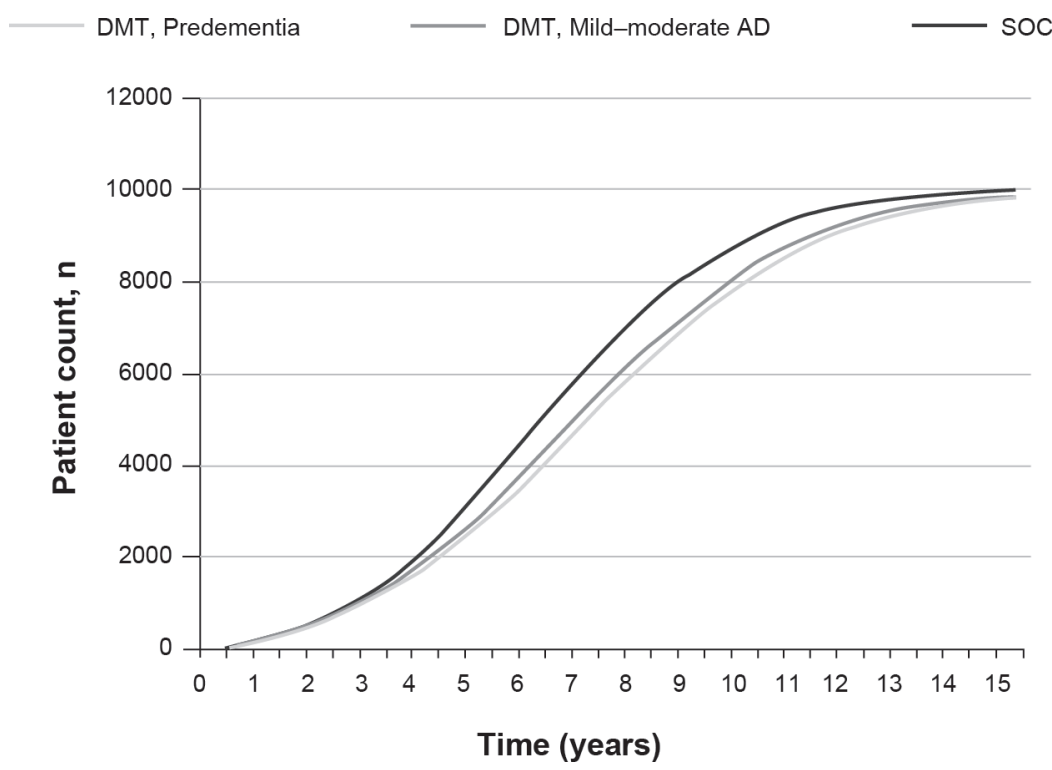

Figure 5 Estimated patient count (y axis) and time of death ( $x$ axis) for patients initiating DMT during predementia AD (light gray line), DMT during mild-moderate AD (medium gray line), or SOC during mild-moderate AD (black line).

Abbreviations: AD, Alzheimer's disease; DMT, disease-modifying therapy; SOC, standard of care.

The strongest finding of this exercise was that, while there was a modest benefit in total life-years gained by treating earlier, the distribution of these years skewed towards milder disease (Figures 3-5). As shown in Figures 3 and 4 and Table 2, from our model, we found that treating predementia Alzheimer's patients with DMTs increased life-years in the predementia to mild Alzheimer's disease states on average from 3.2 years (under standard of care) to 4.0 years (following DMT), while life-years spent in moderate to severe Alzheimer's disease decreased from 2.6 to 2.3 years. This suggests that patients using a DMT with an efficacy of only $25 \%$ greater than the current standard of care (eg, patients treated with donepezil upon reaching mild Alzheimer's disease) can expect a gain of almost 10 months in time spent in milder disease. This also suggests that with increasing efficacy of DMT treatment above this level, it is likely that even greater benefit in terms of time in a milder disease state as well as more relief from moderate to severe disease will be achieved. Also, because patients entered the simulation at 75 years of age, natural mortality is likely to have blunted the benefit of early DMT. Regarding younger patients, it is possible that these results will be even more pronounced given that fewer of the younger, relatively healthier patients die of causes potentially unrelated to Alzheimer's disease.

Our model also revealed that, in addition to the gain in time spent in milder disease, patients with early intervention who were treated with an effective DMT were able to remain at home longer and avoid long-term care compared with patients who receive the standard of care (Figures 6 and 7). The average time remaining in the community increased from 4.4 to 5.4 years, while time in long-term care decreased from 1.3 to 0.9 years. This impact grew larger as the advantage of the DMT increased (Table 3). Thus, as the effectiveness of the drug increased, the overall survival of the patients was increased marginally. However, the balance of time that patients are able to remain at home and out of long-term care was increased, and the amount of time spent living in long-term care decreased. Additionally, because placement in long-term care is a function of disease state and age, earlier intervention in younger

Table 3 Life-years in the community (home) versus in long-term care for different DMT efficacies (all data in years)

\begin{tabular}{|c|c|c|c|c|c|c|}
\hline \multirow[t]{2}{*}{ DMT relative advantage } & \multicolumn{2}{|c|}{ DMT during predementia } & \multicolumn{2}{|c|}{ DMT during moderate AD } & \multicolumn{2}{|c|}{ SOC during moderate $A D$} \\
\hline & Home & LTC & Home & LTC & Home & LTC \\
\hline $10 \%$ & 4.87 & 1.13 & 4.79 & 1.16 & 4.44 & 1.30 \\
\hline $20 \%$ & 5.16 & 1.02 & 4.99 & 1.08 & 4.44 & 1.30 \\
\hline $25 \%$ & 5.37 & 0.90 & 5.10 & 1.04 & 4.44 & 1.30 \\
\hline
\end{tabular}

Abbreviations: AD, Alzheimer's disease; DMT, disease-modifying therapy; LTC, long-term care; SOC, standard of care. 

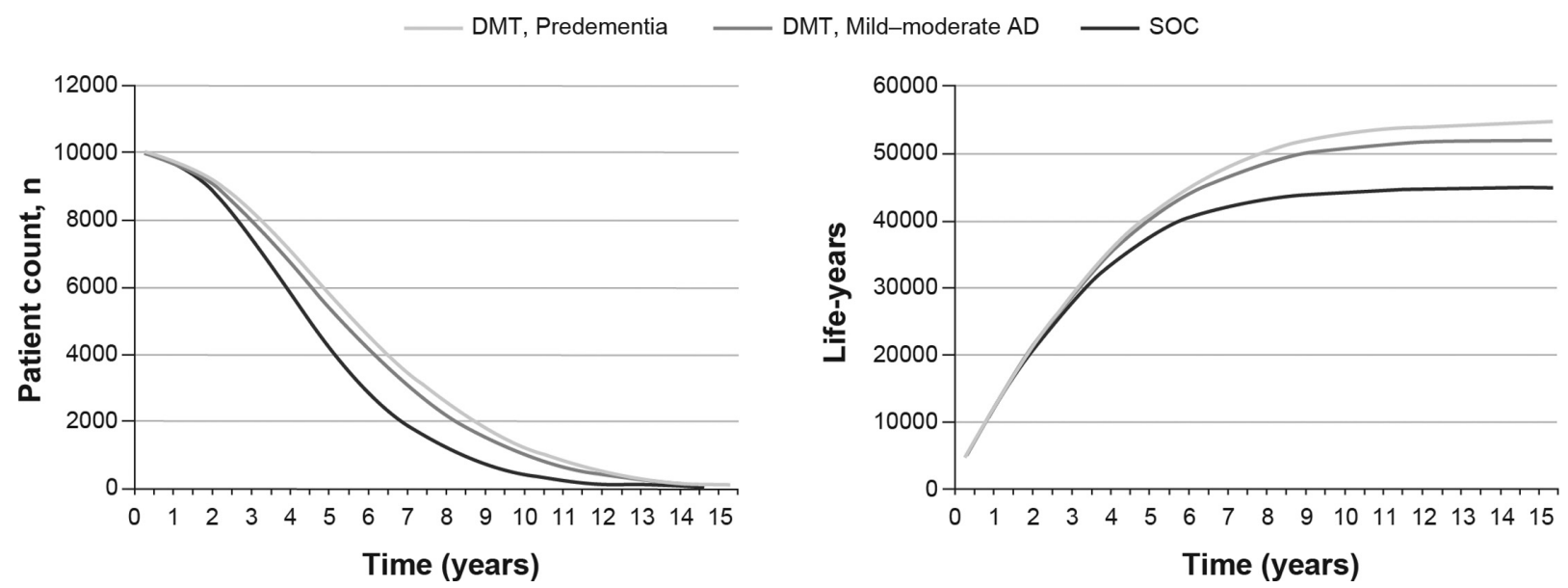

Figure 6 Estimated patient count ( $y$ axis) and time spent ( $x$ axis) in the community if initiating DMT during predementia AD (light gray line), DMT during mild-moderate AD (medium gray line), or SOC during mild-moderate AD (black line). Right panel: the cumulative time spent in the community if initiating DMT during predementia AD (light gray line), DMT during mild-moderate AD (medium gray line), or SOC during mild-moderate AD (black line).

Abbreviations: AD, Alzheimer's disease; DMT, disease-modifying therapy; SOC, standard of care.

patients (under 75 years of age) is also likely to have an even more significant impact on the outcomes of interest.

\section{Conclusion}

This is the first Markov model simulation of its kind to be performed examining the effect of DMT in Alzheimer's disease. Our exercise demonstrated, not unexpectedly, that intervening earlier with potential DMTs would appear to affect the course and outcomes of Alzheimer's disease significantly. Similar results supporting these outcomes have been seen in simulations generated by the Alzheimer's Association in its 2010 report. $^{3}$
Rather than simply delaying the entry into more severe disease states and, ultimately, placement into long-term care, the impact of treatment is to reduce the time spent in these more severe and costly states. Therefore, with only a modest (efficacy 25\%) impact on the course of disease progression, DMTs show promise for prolonging life and shifting the balance in favor of milder disease and more independence for patients with Alzheimer's disease.

\section{Disclosure}

This study was funded by Bristol-Myers Squibb, Princeton, New Jersey.

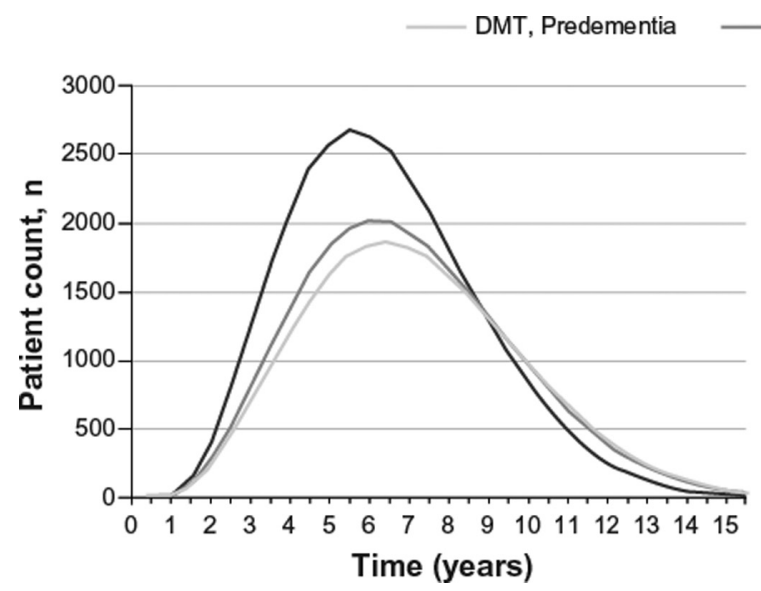

DMT, Mild-moderate AD $\quad$ SOC

Figure 7 Estimated count of patients (y axis) and time spent (x axis) in long-term care if initiating DMT during predementia AD (light gray line), mild-moderate AD (medium gray line), or SOC during predementia AD (black line). Right panel: the cumulative time spent in long-term care if initiating DMT during predementia (light gray line), DMT during mild-moderate $A D$ (medium gray line), or SOC during mild-moderate $A D$ (black line).

Abbreviations: AD, Alzheimer's disease; DMT, disease-modifying therapy; SOC, standard of care. 


\section{References}

1. Cummings JL. Alzheimer's disease. N Engl J Med. 2004;351(1):56-67.

2. Ferri CP, Prince M, Brayne C, et al. Global prevalence of dementia: a Delphi consensus study. Lancet. 2005;366(9503):2112-2117.

3. Alzheimer's Association. 2010 Alzheimer's disease facts and figures. Alzheimers Dement. 2010;6(2):158-194.

4. Sadik K, Wilcock G. The increasing burden of Alzheimer disease. Alzheimer Dis Assoc Disord. 2003;17(Suppl 3):S75-S79.

5. Mitchell AJ, Monge-Argilés JA, Sánchez-Paya J. Do CSF biomarkers help clinicians predict the progression of mild cognitive impairment to dementia? Pract Neurol. 2010;10(4):202-207.

6. Salloway S, Mintzer J, Weiner MF, Cummings JL. Disease-modifying therapies in Alzheimer's disease. Alzheimer Dement. 2008;4(2):65-79.

7. Batemann RJ, Klunk WE. Measuring target effect of proposed diseasemodifying therapies in Alzheimer's disease. Neurotherapeutics. 2008;5(3):381-390.

8. Salloway S, Correia S. Alzheimer disease: time to improve its diagnosis and treatment. Cleve Clin J Med. 2009;76(1):49-57.

9. Landau SM, Harvey D, Madison CM, et al. Comparing predictors of conversion and decline in mild cognitive impairment. Neurology. 2010;75(3):230-238.

10. Andersson C, Blennow K, Johansson S-E, et al. Differential CSF biomarker levels in APOE- $\varepsilon 4$-positive and -negative patients with memory impairment. Dement Geriatr Cogn Disord. 2007;23(2):87-95.

11. Mattson N, Zetterberg H, Hansson O, et al. CSF biomarkers and incipient Alzheimer disease in patients with mild cognitive impairment. JAMA. 2009;302(4):385-393.
12. Blennow K, Zetterberg H. Cerebrospinal fluid biomarkers for Alzheimer's disease. J Alzheimers Dis. 2009;18(2):413-417.

13. Dubois B, Feldman HH, Jacova C, et al. Research criteria for the diagnosis of Alzheimer's disease: revising the NINCDS-ADRDA criteria. Lancet Neurol. 2007;6(8):734-746.

14. Briggs A, Sculpher M. An introduction to Markov modelling for economic evaluation. Pharmacoeconomics. 1998;13(4):397-409.

15. Stewart A, Phillips R, Dempsey G. Pharmacotherapy for people with Alzheimer's disease: a Markov-cycle evaluation of five years' therapy using donepezil. Int J Geriatr Psychiatry. 1998;13(7):445-453.

16. Jack CR, Lowe VJ, Weigand SD; for Alzheimer's Disease Neuroimaging Initiative. Serial PIB and MRI in normal, mild cognitive impairment and Alzheimer's disease: implications for sequence of pathological events in Alzheimer's disease. Brain. 2009;132(Pt 5):1355-1365.

17. Hux MJ, O'Brien BJ, Iskedjian M, Goeree R, Gagnon M, Gauthier S. Relation between severity of Alzheimer's disease and costs of caring. CMAJ. 1998;159(5):457-465.

18. United States statistical abstract 2010. Available from: http://www. census.gov/compendia/statab/2010/2010edition.html. Accessed October $15,2010$.

19. Neumann PJ, Araki SS, Arcelus A, et al. Measuring Alzheimer's disease progression with transition probabilities: estimates from CERAD. Neurology. 2001;57(6):957-964.
ClinicoEconomics and Outcomes Research

\section{Publish your work in this journal}

ClinicoEconomics \& Outcomes Research is an international, peerreviewed open-access journal focusing on Health Technology Assessment, Pharmacoeconomics and Outcomes Research in the areas of diagnosis, medical devices, and clinical, surgical and pharmacological intervention. The economic impact of health policy and health systems

\section{Dovepress}

organization also constitute important areas of coverage. The manuscript management system is completely online and includes a very quick and fair peer-review system, which is all easy to use. Visit http://www.dovepress.com/testimonials.php to read real quotes from published authors. 